Article

\title{
Molecular Docking and Dynamics Simulation of Protein $\beta$-Tubulin and Antifungal Cyclic Lipopeptides
}

\author{
Nubia Noemi Cob-Calan ${ }^{1}$, Luz America Chi-Uluac ${ }^{2}$ (), Filiberto Ortiz-Chi ${ }^{3}{ }^{(1)}$, \\ Daniel Cerqueda-García ${ }^{4}(\mathbb{D})$, Gabriel Navarrete-Vázquez ${ }^{5}$, Esaú Ruiz-Sánchez ${ }^{1, *(D)}$ and \\ Emanuel Hernández-Núñez $4, *$ (D) \\ 1 Tecnológico Nacional de Mexico, Instituto Tecnológico de Conkal, Conkal C.P.97345, Yucatán, Mexico; \\ nubia.cob@itconkal.edu.mx \\ 2 Departamento de Física Aplicada, CINVESTAV-IPN Unidad Mérida, Mérida C.P. 97310, Yucatán, Mexico; \\ chiamerika@hotmail.com \\ 3 CONACYT-Universidad Juárez Autónoma de Tabasco, Centro de Investigación de Ciencia y Tecnología \\ Aplicada de Tabasco, Cunduacán C.P.86690, Tabasco, Mexico; filiberto.ortiz@ujat.mx \\ 4 CONACYT-Departamento de Recursos del Mar, CINVESTAV-IPN Unidad Mérida, Mérida C.P.97310, \\ Yucatán, Mexico; dacegabiol@ciencias.unam.mx \\ 5 Facultad de Farmacia, Universidad Autónoma del Estado de Morelos, Cuernavaca C.P.62209, Morelos, \\ Mexico; gabriel_navarrete@uaem.mx \\ * Correspondence: esau.ruiz@itconkal.edu.mx (E.R.-S.); emanuel.hernandez@cinvestav.mx (E.H.-N.); \\ Tel.: +52-999-912-4135 (ext. 121) (E.R.-S.); +52-999-105-5211 (E.H.-N.)
}

Received: 13 August 2019; Accepted: 15 September 2019; Published: 18 September 2019

\begin{abstract}
To elucidate interactions between the antifungal cyclic lipopeptides iturin A, fengycin, and surfactin produced by Bacillus bacteria and the microtubular protein $\beta$-tubulin in plant pathogenic fungi (Fusarium oxysporum, Colletrotrichum gloeosporioides, Alternaria alternata, and Fusarium solani) in molecular docking and molecular dynamics simulations, we retrieved the structure of tubulin co-crystallized with taxol from the Protein Data Bank (PDB) (ID: 1JFF) and the structure of the cyclic lipopeptides from PubChem (Compound CID: 102287549, 100977820, 10129764). Similarity and homology analyses of the retrieved $\beta$-tubulin structure with those of the fungi showed that the conserved domains shared $84 \%$ similarity, and the root mean square deviation (RMSD) was less than $2 \AA$. In the molecular docking studies, within the binding pocket, residues Pro274, Thr276, and Glu27 of $\beta$-tubulin were responsible for the interaction with the cyclic lipopeptides. In the molecular dynamics analysis, two groups of ligands were formed based on the number of poses analyzed with respect to the RMSD. Group 1 was made up of 10, 100, and 500 poses with distances 0.080 to $0.092 \mathrm{~nm}$ and RMSDs of 0.10 to $0.15 \mathrm{~nm}$. For group 2, consisting of 1000 poses, the initial and final distance was $0.1 \mathrm{~nm}$ and the RMSDs were in the range of 0.10 to $0.30 \mathrm{~nm}$. These results suggest that iturin $A$ and fengycin bind with higher affinity than surfactin to $\beta$-tubulin. These two lipopeptides may be used as lead compounds to develop new antifungal agents or employed directly as biorational products to control plant pathogenic fungi.
\end{abstract}

Keywords: molecular dynamics; molecular docking; $\beta$-tubulin; cyclic lipopeptides; antifungal activity

\section{Introduction}

Lipopeptides are a class of proteins with antibiotic properties against a wide range of microorganisms, including plant pathogenic fungi [1]. They consist of a lipid tail linked to a short oligopeptide that can be linear or cyclic [2]. The ring of the cyclic lipopeptides is formed by an 
ester (lactone) or amide (lactam) group. Cyclic lipopeptides are produced as secondary metabolites by a variety of bacterial genera, including Actinomycetes, Streptomyces, Bacillus, and Pseudomonas [2-4].

Cyclic lipopeptides produced by Bacillus are classified into three categories: iturins, fengycins, and surfactins $[1,2,5]$. They are all fungicidal against various species of plant pathogenic fungi within the groups Deuteromycota, Basidiomycota, and Ascomycota [6-8]. The antifungal action includes the damage and dysfunction of membranes and organelles, alterations in mitochondrial membrane potential, oxidative stress, and chromatin condensation [8]. Although the membrane damage caused by permeabilization and ion-conducting pore formation in the cell membrane has been characterized in detail $[9,10]$, other target sites of these compounds have been scarcely studied. In this context, the $\beta$-tubulin protein may be a suitable target site, because it has been highly implicated as the target of a wide range of synthetic fungicides [11,12]. Fungicides act on $\beta$-tubulin by inhibiting the assembly of heterodimers of $\alpha$ - and $\beta$-tubulin into microtubules, which are vital to various cellular processes such as cell signaling, cell motility, cell division, and mitosis [13]. $\beta$-tubulin-targeting agents may bind to the protein at any of four sites: laulimalide, taxane/epothilone, vinca alkaloid, and colchicine sites [11,12]. These binding pockets interact with active compounds based on the hydrophobicity and size of the molecule. Two binding pockets, taxane/epothilone and vinca alkaloid, interact with large and complex molecules, opening new avenues to explore large compounds as potential $\beta$-tubulin target agents [11,12]. Compounds that bind taxane/epothilone are structurally and chemically similar to the cyclic lipopeptides in terms of the number of carbons in the ring [14-16].

The search for new candidate target molecules, including $\beta$-tubulin, in pathogenic fungi and other pathogens of plants has become more efficient with the aid of computer-simulated molecular docking studies. For example, in the fungus Fusarium oxysporum, 2,4-di-tert-butylphenol, a compound that effectively inhibits spore germination and hyphal growth in vitro, was discovered through molecular docking analyses to potentially bind to $\beta$-tubulin protein at the HIS 118 and THR 117 residues [17]. In the plant parasitic nematode Bursaphelenchus xylophilus, the compounds amocarzine, mebendazole, and flubendazole were revealed to bind various target sites, including $\beta$-tubulin, by analyzing hypothetical binding sites in a protein model constructed through molecular docking [18].

Here, we used in silico studies of the molecular interaction of the cyclic lipopeptides iturin A, fengycin or surfactin with $\beta$-tubulin to identify which molecules have the highest affinity for binding $\beta$-tubulin from four plant pathogenic fungi. Modified $\beta$-tubulin was stabilized and validated. Coupling studies of the cyclic lipopeptides showed that all three can potentially bind to $\beta$-tubulin; that iturin $\mathrm{A}$ and fengycin bind with higher affinity than surfactin and are the most promising antifungal candidates.

\section{Results and Discussion}

\subsection{Homology Modeling of $\beta$-Tubulin}

The search for homologs of the $\beta$-tubulin template using the protein Basic Local Alignment Search Tool (blastp) yielded positive hits with $84 \%$ similarity for a peptide sequence from four species of fungi: F. oxysporum, Colletrotrichum gloeosporioides, Alternaria alternata, and Fusarium solani obtained from PubChem. Analysis of these sequences with HMMER software identified conserved domains of the protein families of tubulin (FtsZ amino acids 3-212) and tubulin C (amino acids 261-382) with an e-value $<7 \mathrm{e}^{-42}$ (Figure 1 and Table S2). Hidden Markov models implemented in the HMMER3 software are robust and have been used for remote protein homology detection [19]; thus, with the results of the HMMER analysis, we confirmed that the template protein and the proteins of the four species of fungi are homologous. In addition, we also found that the catalytic site (where taxol binds as a ligand) was constant in the protein $\beta$-tubulin of the four fungal species analyzed. In a similar study, De Pereda et al. [20] found similarity among several tubulins, with the following proportions: $33 \%$ for $\alpha, 21 \%$ for $\beta$, and $45 \%$ for other secondary structures. Carpenter et al. [21] evaluated 475 tubulin isotypes, finding global and structural similarities, but differences among net electric charges, 
solvent accessible surface areas, and electrical dipole moments. They concluded that these differences are responsible for the variations in their cellular activity. In a blastp search using tubulin from the nematode Bursaphelenchus xylophilus as the query, the X-ray crystal structure of ID 5IJ0 in the Protein Data Bank (PDB) was identified as homologous and shared $86 \%$ similarity with the nematode tubulin [18].

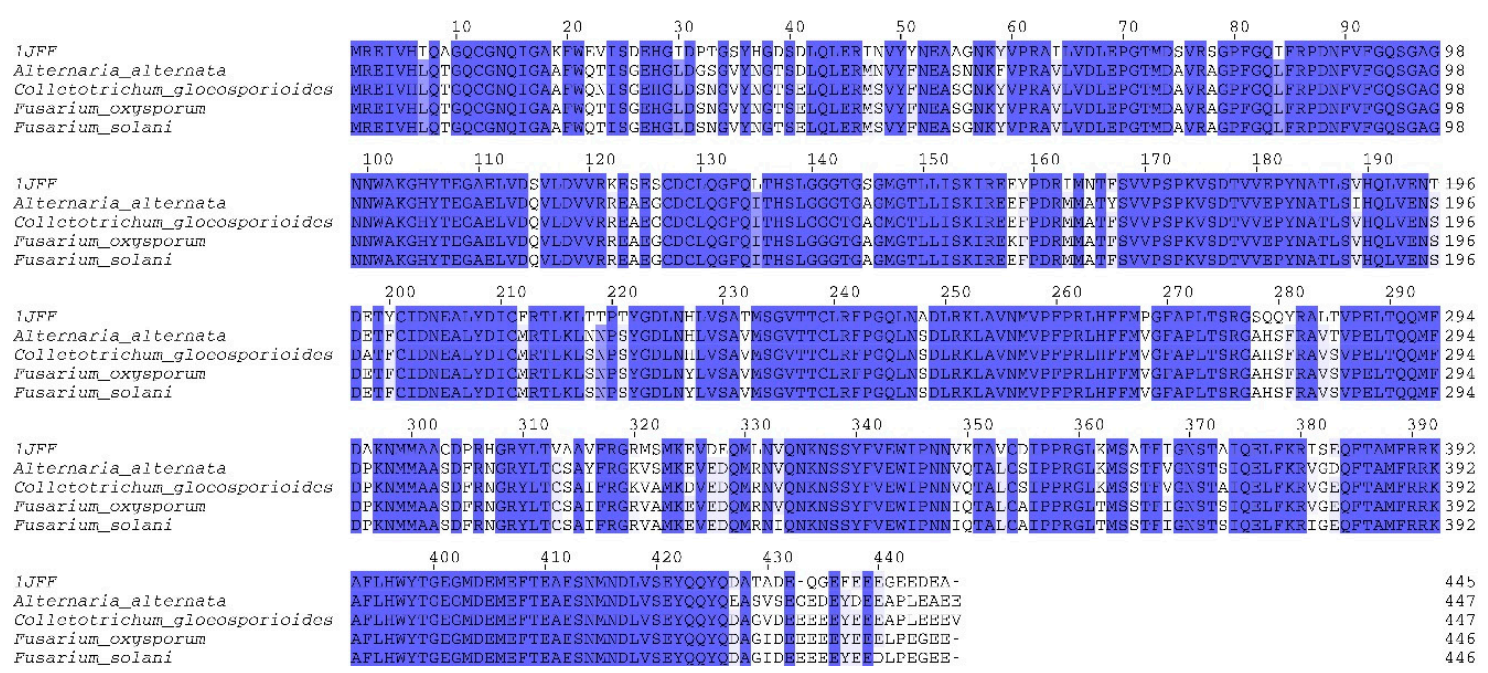

Figure 1. Alignment of the peptide sequence from the four fungal species with the template sequence of $\beta$-tubulin (1JFF) obtained from the PubChem. Highly conserved regions are indicated in blue.

\subsection{Active Site Validation}

The active site of $\beta$-tubulin was validated with the co-crystallized native ligand taxol. Comparison of the poses obtained by of the AutoDock Vina program against those of the crystallized protein yielded root mean square deviation $(\mathrm{RMSD})=1.56 \AA$, indicating an appropriate optimization score $[22,23]$. These values are small and support binding at the simulation site with the original orientation of the co-crystallized molecule (Figure 2).

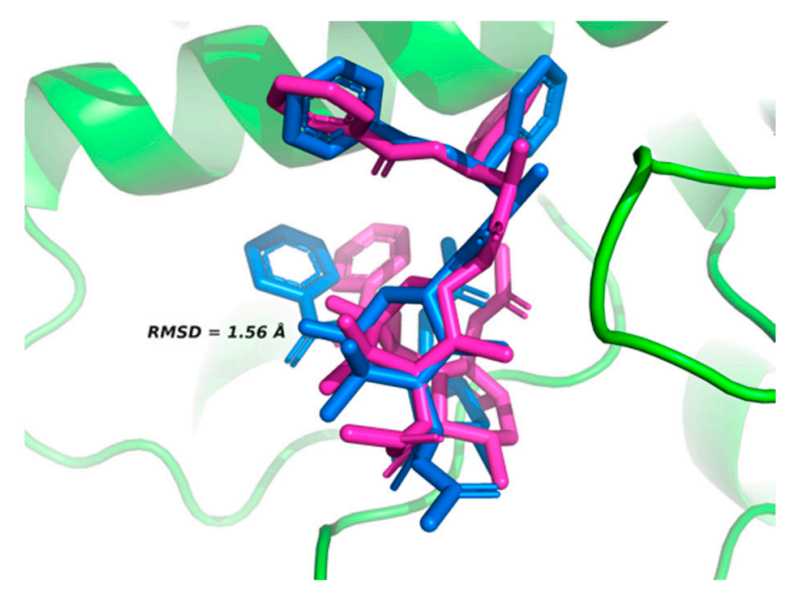

Figure 2. Ligand-binding site of $\beta$-tubulin protein with co-crystalized native taxol (blue) and taxol as posed by the Autodock Vina program (pink).

\subsection{Molecular Docking Analysis}

In the analyses to explore the binding affinities of the three-antifungal cyclic lipopeptides with the potential target site in $\beta$-tubulin, iturin A, fengycin, and surfactin exhibited docking interactions with $\beta$-tubulin. Figure 3 shows the optimized structures of these compounds, using a stochastic global conformational search strategy interfaced to the Gaussian 16 code [24]. 


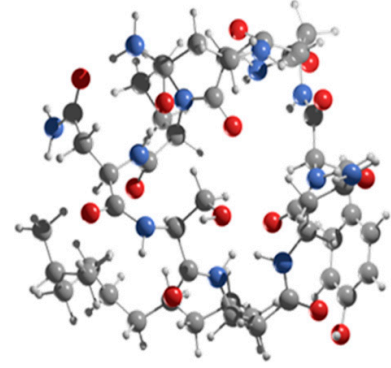

(a)

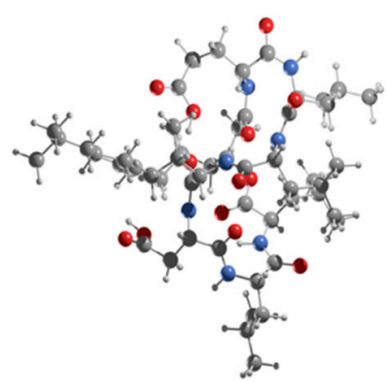

(b)

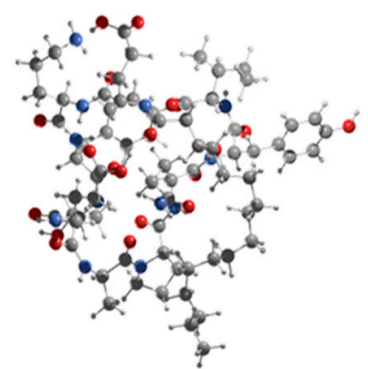

(c)

Figure 3. Three-dimensional structural representation of (a) iturin A, (b) fengycin, and (c) surfactin calculated by a homemade code interfaced to Persistence of Vision Ray-tracer (POVRAY.)

Docking of the alkaloid taxol, used as the native ligand, with the modeled $\beta$-tubulin protein was simulated using the AutoDock Vina open source program and a genetic algorithm. To explore the binding affinity and the molecular basis of the interactions, the three lipopeptides were docked within the binding sites predicted from the $\beta$-tubulin model. The evolutionary metaheuristic used showed that iturin $\mathrm{A}$, fengycin, and surfactin exhibited docking poses with high binding affinities (in terms of coupling energy). The binding energies and amino acid interactions for each compound with $\beta$-tubulin are given in Table 1 . The docking analysis of the compounds with $\beta$-tubulin generated negative values for free energy, suggesting high affinity for the binding pocket. Although all the binding conformations of each compound in the active binding pocket involved both H-bond and non-bonded interactions, iturin $\mathrm{A}$ and fengycin had higher binding affinities for $\beta$-tubulin based on lower binding energies.

Table 1. Binding affinity for the molecular coupling in the $\beta$-tubulin protein complex with taxol and the test lipopeptides.

\begin{tabular}{|c|c|c|c|c|c|c|}
\hline Compound & $\begin{array}{l}\text { No. of } \\
\text { H-Bonds }\end{array}$ & $\begin{array}{l}\text { Residue } \\
\text { Receptor }\end{array}$ & Ligand & $\begin{array}{c}\text { Bond } \\
\text { Length (Å) }\end{array}$ & $\begin{array}{l}\text { Docking Score } \\
\text { (kcal/mol) }\end{array}$ & $k_{i}\left(\mu \mathbf{M}^{)}\right.$ \\
\hline Taxol & 1 & Thr276(N) & O06 & 2.92 & -9.1 & 0.202 \\
\hline \multirow[t]{2}{*}{ Iturin A } & \multirow[t]{2}{*}{2} & $\begin{array}{l}\text { Pro274(O) } \\
\text { Thr276(O) }\end{array}$ & $\begin{array}{l}\text { (ND2)68 } \\
\text { (ND2)66 }\end{array}$ & $\begin{array}{l}2.38 \\
2.16\end{array}$ & \multirow[t]{2}{*}{-7.0} & \multirow[t]{2}{*}{7.084} \\
\hline & & Glu27(OE2) & (O)114 & 2.11 & & \\
\hline \multirow[t]{2}{*}{ Fengycin } & \multirow[t]{2}{*}{3} & Thr276(O) & (N)115 & 2.43 & \multirow[t]{2}{*}{-7.0} & \multirow[t]{2}{*}{7.084} \\
\hline & & Thr276(OG1) & (N)115 & 2.09 & & \\
\hline Surfactin & 1 & His229(NE2) & $\mathrm{H}$ & 2.7 & -6.3 & 23.188 \\
\hline
\end{tabular}

The binding modes of iturin A, fengycin, and surfactin within the binding site of $\beta$-tubulin are important for the design of highly toxic compounds against plant pathogenic fungi. The binding interactions of these compounds with the active site are stabilized through H-bonds and non-bonded interactions (Figures S1, S2, and S3). Generally, a non-bonded interaction (e.g., van der Waal interaction) contributes to a more stable protein-ligand complex and thus greater antimicrobial activity [26]. From the docking results, the binding energy for $\beta$-tubulin with iturin $A(-7.0 \mathrm{kcal} / \mathrm{mol})$ is equal to that for $\beta$-tubulin with fengycin $(-7.0 \mathrm{kcal} / \mathrm{mol})$. The binding energy for $\beta$-tubulin with surfactin was $-6.3 \mathrm{kcal} / \mathrm{mol}$ (Figure 4). The Ki value for the binding of taxol with $\beta$-tubulin was lower $(K i=0.20 \mu \mathrm{M})$ compared to those observed for the binding of iturin A or fengycin with $\beta$-tubulin $(K i=7.084 \mu \mathrm{M}$ for each). The $K i$ value for the binding of surfactin with $\beta$-tubulin was high $(K i=23.18 \mu \mathrm{M})$, indicating that this cyclic lipopeptide had the lowest affinity of the three (Table 1). 


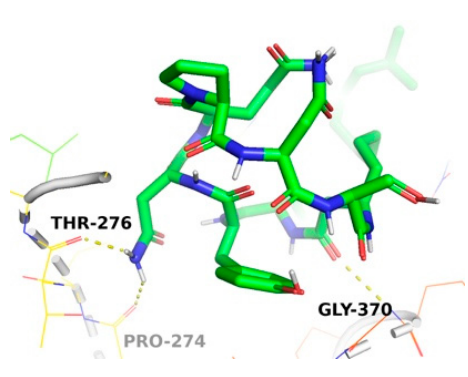

(a)

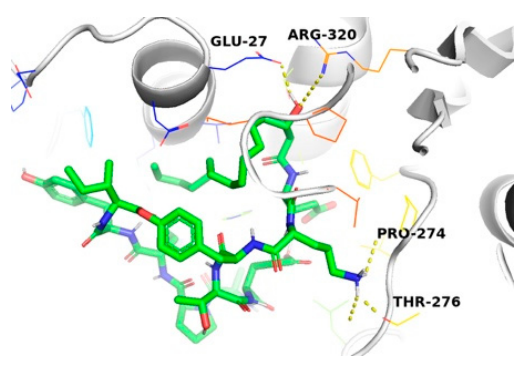

(b)

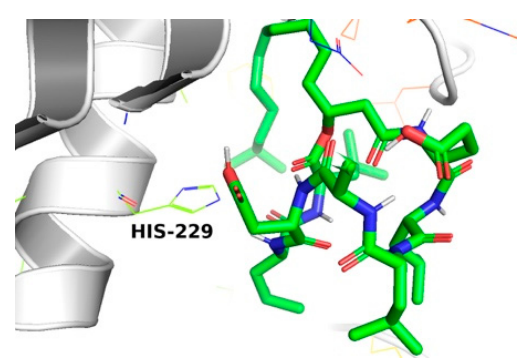

(c)

Figure 4. Molecular docking simulation showing the interaction of (a) iturin A, (b) fengycin, and (c) surfactin (green and blue) with active site residues of $\beta$-tubulin (grey).

The docking analysis revealed that iturin $\mathrm{A}$ and fengycin have equal binding affinity and higher than surfactin for $\beta$-tubulin based on the docking energy and number of $\mathrm{H}$-bonds. Iturin $\mathrm{A}$ binds to $\beta$-tubulin with two H-bonds, and to fengycin with three (Table 1). For iturin A, the amino acid in position 68 forms an H-bond with the oxygen of Pro274 of $\beta$-tubulin with a bond length of $2.38 \AA$. Similarly, another hydrogen bond forms between the amino acid in position 66 of iturin A and the oxygen of Thr276 of $\beta$-tubulin with a bond length of $2.16 \AA$. For fengycin, the oxygen in position 114 forms an H-bond with the oxygen of Glu27 and Thr276 of $\beta$-tubulin with a bond length of $2.11 \AA$. The second and third H-bonds are formed between the nitrogen at position 115 of the fengycin, which connects with the oxygen and carbonyl of the Thr276 of $\beta$-tubulin (bond lengths of 2.43 and $2.09 \AA$, respectively). All details of the atoms involved in bonding with ligands, bond lengths, docking energies, and Ki values are given in Table 1.

Hypothetically, the higher the affinity of a ligand for its target protein, the more effective its activity at the cellular or organism level. In that case, iturin A and fegycin would be more toxic than surfactin. In agreement with this assumption, experiments with plant pathogenic fungi showed that iturin A and fengycin were more effective than surfactin at inhibiting mycelial growth and reducing the severity of leaf damage $[27,28]$.

Again, in a hypothetical interaction between iturin A or fengycin with $\beta$-tubulin in plant pathogenic fungi, cellular functions such as spore germination and hyphal growth needed for pathogenesis would be impaired due to the inhibition of microtubule formation or an alteration in microtubule stability [11]. Iturin A and fengycin may thus have great potential as biorational fungicides. However, further evaluation of the effectiveness of these lipopeptides for disease control is required.

It is important to note that, based on the results of the present study, we cannot establish the specific mechanism of action of the lipopeptides on $\beta$-tubulin or classify them as either microtubule stabilizers or destabilizers. Further studies are necessary to assess their impact on dimer stability and their toxicity.

\subsection{Molecular Dynamics (MD)}

To assess the stability of the $\beta$-tubulin-iturin A, $\beta$-tubulin-fengycin, and $\beta$-tubulin-surfactin complexes, we used the following descriptors: ligand RMSD, interface RMSD (IRMSD), number of hydrogen bonds, and distance between the center of mass of the binding site and the ligand.

The ligand RMSD is displayed in Figure 5a. The ligand backbone RMSD was estimated using the structure in the equilibrated stage of the simulation (frame at $50 \mathrm{~ns}$ ) as a reference. The ligand RMSDs remained below $2.5 \AA$, with the fengycin backbone RMSD being the smallest and the most stable (smallest deviation in the RMSD).

The interface RMSD (IRMSD) is shown in Figure 5b. Because the IRMSD includes all the amino acids in the protein that are closest to the ligand (less than $4 \AA$ from the ligand), it can give insight into the stability of the interacting amino acids. All IRMSDs were below $3 \AA$, while surfactin and iturin A had the lowest IRMSD values, indicating the highest stability. 


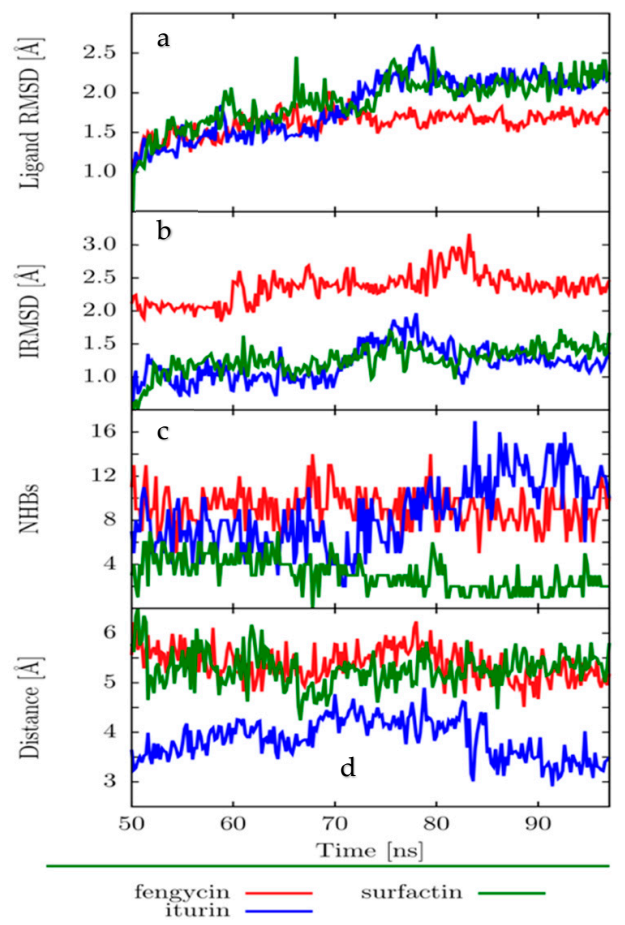

Figure 5. Time evolution of (a) the ligand root means square deviation (RMSD), (b) the interface RMSD (IRMSD), (c) the number of H-bonds between the protein and ligand, and (d) the distance between the ligand and tubulin-binding site. Analysis was performed by Gromacs.

The number of H-bonds is given in Figure 5c. Iturin A and fengycin had the most $\mathrm{H}$-bonds, but the fengycin H-bonds were more stable over the entire simulation and would likely play a significant role in stabilizing the protein-ligand interactions. The H-bonds in docking structures were maintained during the Molecular Dynamics (MD) simulations; in addition, other H-bonds were observed.

The analysis of the distance between the center of the mass of the protein and the center of the mass of each ligand showed that the shortest distance was found for iturin A (Figure 5d).

The protein-ligand complexes formed are thermally stable at $300 \mathrm{~K}$. Ligands do not tend to detach over $100 \mathrm{~ns}$ of simulation in a realistic solvent environment, depending on the distance, the number of $\mathrm{H}$-bonds, and the IRMSD and ligand RMSD analyses. We conclude that iturin A and fengycin were more stable over the simulation.

\section{Materials and Methods}

\subsection{Domain Identification and Template Search}

The sequence of the crystalized $\beta$-tubulin protein was downloaded from the Protein Data Bank (ID:1JFF was used as a template) and used as the search query in the online platform of blastp [29] to find homologous protein sequences for the phytopathogenic fungi Fusarium oxysporum (XP_018241948.1), Colletrotrichum gloeosporioides (XP_ELA34262.1), Alternaria alternata (XP_018387617.1), and Fusarium solani (XP_003046201.1). The homologous proteins that were identified by significant hits (similarity > $70 \%$, e-value $<1 \mathrm{e}^{-10}$ ) were then used in a search against the Pfam database for conserved domains of the $\beta$-tubulin family using the software HMMER v3.2.1.

\subsection{Conformational Search of the Lipopeptides}

Initial structures for iturin A (CID:102287547), fengycin (CID:100977820), and surfactin (CID:10129764) were obtained from the PubChem database (https://pubchem.ncbi.nlm.nih.gov). These molecules have a high number of degrees of torsional freedom, so the global conformational search for their rotational isomers was performed in two stages. In the first stage, a stochastic algorithm 
generated $10^{4}$ rotational isomers, all dissimilar to each other. All the generated structures were optimized at the semi-empirical level PM6-D3 [30,31], as implemented in the MOPAC code [32]. In the second stage, the best $10^{3}$ energetically favorable rotational isomers were chosen to be fully optimized at the Density Functional Theory (DFT) level through the Gaussian 16 code [24]. The GGA hybrid functional B3LYP [33] was chosen in conjunction with the 6-31G(d) basis set [34], usually used for organic molecules.

\subsection{Validation of Active Site}

The active site on $\beta$-tubulin was validated using taxol as the native ligand (PDB: 1JFF). RMSD was set to less than $2 \AA$ to determine the best docking position between $\beta$-tubulin and the ligands using Autodock Vina [35]. The validation was performed with 10 poses, each in triplicate, selecting the lowest energy value. Protein visualization and overlap were carried out using Pymol 3.1 (Schrödinger, San Diego; http://www.pymol.org/).

\subsection{Molecular Docking}

The docking of $\beta$-tubulin with each ligand (iturin A, fengycin, and surfactin) was simulated using the program AutoDock Vina, which has been used to estimate the conformation of protein-ligand complexes [35] and significantly improves the average accuracy of the binding mode predictions.

All calculations for protein-fixed ligand-flexible docking were analyzed using the Lamarckian Genetic Algorithm (LGA) method [22]. The docking site on $\beta$-tubulin was defined by establishing a grid box using Pymol 3.1 (Table S3). The grid box size for the coordinates $x, y$, and $z$ was $30 \AA$, with a grid spacing of $0.375 \AA$, centered on $x=-2.265, y=7.164$, and $z=17.568 \AA$. The best conformation was chosen based on the lowest binding energy after the docking search was completed. Autodock Vina was set for 1000 modes and exhaustiveness 1000 (exhaustiveness of the global search, roughly proportional to time) for each ligand structure, and for each run, the best pose was saved. The average binding energy for the best poses was used as the final binding energy value. This process was repeated three times.

\subsection{Molecular Dynamics}

For each protein-ligand system, four poses with minimum energy were evaluated $(10,100,500$, 1000 ) with three replicas each (R1, R2, R3). Systems were previously minimized; the number of particles, volume, and temperature (NVT) as well as the number of particles, pressure, and temperature (NPT) were equilibrated; then, MD simulations in water over $100 \mathrm{~ns}$ were run.

All calculations (minimization, NVT equilibration, NPT equilibration, and MD simulations) were performed using GROMACS 5.1.2 [36] with GPU support. We used the GROMOS96 43A1 force field for the protein and the ligand in all cases. We used the simple point-charge (SPC)model for water molecules. The molecules were solvated in a dodecahedron box, with periodic boundary conditions and a minimum distance between the solute and the box of $1.5 \mathrm{~nm}$. Sodium and chloride ions were added to neutralize the system. Energy minimization steps were carried out using the steepest descent algorithm. During NVT ensemble simulations, harmonic position restraints were applied to the solute heavy atoms with a force constant of $1000 \mathrm{~kJ} \mathrm{~mol}^{-1} \mathrm{~nm}^{-2}$. The MD production runs were performed using 2 fs as the time step. For pressure coupling, we used Berendsen at 1 bar. Temperature was controlled by setting the Langevin dynamics at $300 \mathrm{~K}$. Standard $x y z$ periodic boundary conditions were considered. A cut-off distance of $1.0 \mathrm{~nm}$ for the Coulomb and van der Waals neighbor list was updated according to the Verlet cut-off scheme. The long-range part of the Coulomb interactions was evaluated using the particle-mesh Ewald (PME) method with a relative tolerance of 5-10, order of 6, and Fourier spacing of 0.1. All bonds were constrained using linear constraint solver for molecular simulations (LINCS), while SETTLE was used for constraining the water molecules.

For the analysis of MD simulations, we assessed the ligand root mean square deviation (RMSD), interface root means square deviation (IRMSD), number of H-bonds, and distances between the 
protein-binding sites and the ligand. The ligand RMSD was calculated for the backbone using an equilibrated frame at $50 \mathrm{~ns}$ as a reference. For the IRMSD, we first aligned the backbone protein trajectory to the backbone of the crystal structure. Then, we grouped clusters by similar conformations of the MD trajectory and chose the middle structure of the largest cluster as a representative element. We then found the closest amino acids to the ligand (less than $4 \AA$ ) of these representative structures and calculated the RMSD of those amino acids over the complete $100 \mathrm{~ns}$ MD simulation. We also calculated the distances between the backbone center of mass of the protein-binding site and the backbone center of mass of each ligand. We considered the first $50 \mathrm{~ns}$ of the simulation as an equilibration period, after which we presented the results over the last $50 \mathrm{~ns}$ of MD simulation.

\section{Conclusions}

In conclusion, using molecular docking and molecular dynamics simulation, we demonstrated the interactions of the cyclic lipopeptides iturin A, fengycin, and surfactin with $\beta$-tubulin. The analysis of molecular docking showed that residues Pro274, Thr276, and Glu27 of $\beta$-tubulin are responsible for the formation of hydrogen bonds. Iturin A and fengycin had higher binding affinity than surfactin to the catalytic site of $\beta$-tubulin. These molecules can potentially target $\beta$-tubulin in plant pathogenic fungi. These cyclic lipopeptides can either be tested directly as biorational fungicides or used as lead compounds, thus opening new avenues to biorationally design antifungal agents.

Supplementary Materials: Supplementary Materials are available online. Table S1. PubChem accessions of $\beta$-tubulin in fungi with highest homology to template $\beta$-tubulin. Table S2: Conserved domains in $\beta$-tubulin from fungal species tested. Table S3: Validation of active site of $\beta$-tubulin (PDB $=1 \mathrm{JFF}$ ) with taxol as a ligand using the AutoDock Vina program. Table S4: Binding affinity for the molecular coupling in the $\beta$-tubulin protein complexes with taxol and the test lipopeptides. Figure S1: Schematic of molecular docking between $\beta$-tubulin and iturin A at the active site. Figure S2: Schematic of molecular docking between $\beta$-tubulin and fengycin at the active site. Figure S3: Schematic of molecular docking between $\beta$-tubulin and surfactin at the active site.

Author Contributions: N.N.C.-C. and E.H.-N. performed and analyzed the in silico refined molecular docking simulations and wrote the manuscript; E.R.-S. supervised the analysis and wrote the manuscript; F.O.-C. and N.N.C.-C. analyzed the data; L.A.C.-U. and N.N.C-C. analyzed the molecular dynamics data; D.C.-G. and G.N.-V. carried out the validation and formal analysis and revised the drafts.

Funding: This work was supported by CONACYT (CB-2015-01) 254321.

Acknowledgments: The authors acknowledge CINVESTAV for providing the HPC LANCAD CINVESTAV node and ABACUS. The HPC Juchiman (CONACyT, U0003-2015-7-26609) allocated computing resources and contributed to the PFCE-UJAT-2018 project. Financial support was also provided by PRODEP (ITESCAM-003) through the "Conventional support for postgraduate studies", which helped support the research reported here. This work was also supported in part by CONACYT (CB-2015-01) 254321.

Conflicts of Interest: All authors declare no conflict of interest.

\section{References}

1. Ongena, M.; Jacques, P. Bacillus lipopeptides: Versatile weapons for plant disease biocontrol. Trends Microbiol. 2008, 16, 115-125. [CrossRef]

2. Schneider, T.; Müller, A.; Miess, H.; Gross, H. Cyclic lipopeptides as antibacterial agents-Potent antibiotic activity mediated by intriguing mode of actions. Int. J. Med. Microbiol. 2014, 304, 37-43. [CrossRef]

3. Eswari, J.S.; Anand, M.; Venkateswarlu, C. Optimum culture medium composition for lipopeptide production by Bacillus subtilis using response surface model-based ant colony optimization. Sadhana 2016, 41, 55-65. [CrossRef]

4. Raaijmakers, J.M.; de Bruijn, I.; de Kock, M.J.D. Cyclic lipopeptide production by plant-associated Pseudomonas spp.: Diversity, activity, biosynthesis, and regulation. Mol. Plant Microbe Interact. 2006, 19, 699-710. [CrossRef]

5. Ongena, M.; Jacques, P.; Touré, Y.; Destain, J.; Jabrane, A.; Thonart, P. Involvement of fengycin-type lipopeptides in the multifaceted biocontrol potential of Bacillus subtilis. Appl. Microbiol. Biotechnol. 2005, 69, 29-38. [CrossRef] 
6. Chan, Y.K.; Savard, M.E.; Reid, L.M.; Cyr, T.; McCormick, W.A.; Seguin, C. Identification of lipopeptide antibiotics of a Bacillus subtilis isolate and their control of Fusarium graminearum diseases in maize and wheat. BioControl 2009, 54,567-574. [CrossRef]

7. Romero, D.; de Vicente, A.; Olmos, J.L.; Dávila, J.C.; Pérez-García, A. Effect of lipopeptides of antagonistic strains of Bacillus subtilis on the morphology and ultrastructure of the cucurbit fungal pathogen Podosphaera fusca. J. Appl. Microbiol. 2007, 103, 969-976. [CrossRef]

8. Meena, K.R.; Kanwar, S.S. Lipopeptides as the antifungal and antibacterial agents: Applications in food safety and therapeutics. BioMed Res. Int. 2015, 2015, 473050. [CrossRef]

9. Grau, A.; Gómez Fernández, J.C.; Peypoux, F.; Ortiz, A. A study on the interactions of surfactin with phospholipid vesicles. Biochim. Biophys. Acta Biomembr. 1999, 1418, 307-319. [CrossRef]

10. Heerklotz, H.; Seelig, J. Leakage and lysis of lipid membranes induced by the lipopeptide surfactin. Eur. Biophys. J. 2007, 36, 305-314. [CrossRef]

11. Chatterji, B.P.; Jindal, B.; Srivastava, S.; Panda, D. Microtubules as antifungal and antiparasitic drug targets. Expert Opin. 2011, 21, 167-186. [CrossRef] [PubMed]

12. Downing, K.H. Structural Basis for the interaction of tubulin with proteins and drugs that affect microtubule dynamics. Annu. Rev. Cell Dev. Biol. 2000, 16, 89-111. [CrossRef] [PubMed]

13. Vindya, N.G.; Sharma, N.; Yadav, M.; Ethiraj, K.R. Tubulins-The target for anticancer therapy. Curr. Top. Med. Chem. 2015, 15, 73. [CrossRef]

14. St. George, M.; Ayoub, A.T.; Banerjee, A.; Churchill, C.D.M.; Winter, P.; Klobukowski, M.; Cass, C.E.; Ludueña, R.F.; Tuszynski, J.A.; Damaraju, S. Designing and testing of novel Taxanes to probe the highly complex mechanisms by which Taxanes bind to microtubules and cause cytotoxicity to cancer cells. PLoS ONE 2015, 10, e0129168. [CrossRef] [PubMed]

15. Lone, M.Y.; Athar, M.; Manhas, A.; Jha, P.C.; Bhatt, S.; Shah, A. In Silico exploration of Vinca domain tubulin inhibitors: A combination of 3D-QSAR-Based pharmacophore modeling, docking and molecular dynamics simulations. Chem. Sel. 2017, 2, 10848-10853. [CrossRef]

16. Oxberry, M.E.; Geary, T.G.; Prichard, R.K. Assessment of benzimidazole binding to individual recombinant tubulin isotypes from Haemonchus contortus. Parasitology 2001, 122, 683-687. [CrossRef] [PubMed]

17. Dharni, S.; Maurya, A.; Samad, A.; Srivastava, S.K.; Sharma, A.; Patra, D.D. Purification, characterization, and in Vitro activity of 2,4-Di- tert -butylphenol from Pseudomonas monteilii PsF84: Conformational and molecular docking studies. J. Agric. Food Chem. 2014, 62, 6138-6146. [CrossRef] [PubMed]

18. Shanmugam, G.; Lee, S.; Jeon, J. Identification of potential nematicidal compounds against the pine wood nematode, Bursaphelenchus xylophilus through an In Silico Approach. Molecules 2018, 23, 1828. [CrossRef] [PubMed]

19. Johnson, L.S.; Eddy, S.R.; Portugaly, E. Hidden Markov model speed heuristic and iterative HMM search procedure. BMC Bioinform. 2010, 11, 431. [CrossRef]

20. De Pereda, J.M.; Leynadier, D.; Evangelio, J.A.; Chacón, P.; Andreu, J.M. Tubulin Secondary Structure Analysis, Limited Proteolysis Sites, and Homology to FtsZ. Biochemistry 1996, 35, 14203-14215. [CrossRef]

21. Carpenter, E.J.; Huzil, J.T.; Ludueña, R.F.; Tuszynski, J.A. Homology modeling of tubulin: Influence predictions for microtubule's biophysical properties. Eur. Biophys. J. 2006, 36, 35-43. [CrossRef] [PubMed]

22. Herowati, R.; Widodo, G.P. Molecular Docking studies of chemical constituents of Tinospora cordifolia on glycogen phosphorylase. Procedia Chem. 2014, 13, 63-68. [CrossRef]

23. Kumar, P.N.; Swapna, T.H.; Khan, M.Y.; Daddam, J.R.; Hameeda, B. Molecular dynamics and protein interaction studies of lipopeptide (Iturin A) on $\alpha$-amylase of Spodoptera litura. J. Theor. Biol. 2017, 415, 41-47. [CrossRef] [PubMed]

24. Frisch, M.J.; Trucks, G.W.; Schlegel, H.B.; Scuseria, G.E.; Robb, M.A.; Cheeseman, J.R.; Scalmani, G.; Barone, V.; Petersson, G.A.; Nakatsuji, H.; et al. Gaussian 16, Revision C.01. Fox; Gaussian, Inc.: Wallingford, CT, USA, 2016.

25. González-Trujano, M.E.; Uribe-Figueroa, G.; Hidalgo-Figueroa, S.; Martínez, A.L.; Déciga-Campos, M.; Navarrete-Vazquez, G. Synthesis and antinociceptive evaluation of bioisosteres and hybrids of naproxen, ibuprofen and paracetamol. Biomed. Pharmacother. 2018, 101, 553-562. [CrossRef] [PubMed]

26. Sharma, O.P.; Pan, A.; Hoti, S.L.; Jadhav, A.; Kannan, M.; Mathur, P.P. Modeling, docking, simulation, and inhibitory activity of the benzimidazole analogue against $\beta$-tubulin protein from Brugia malayi for treating lymphatic filariasis. Med. Chem. Res. 2012, 21, 2415-2427. [CrossRef] 
27. Kim, P.I.; Ryu, J.; Kim, Y.H.; Chi, Y.T. Production of biosurfactant lipopeptides Iturin A, fengycin and surfactin A from Bacillus subtilis CMB32 for control of Colletotrichum gloeosporioides. J. Microbiol. Biotechnol. 2010, 20, 138-145.

28. Li, Y.; Héloir, M.; Zhang, X.; Geissler, M.; Trouvelot, S.; Jacquens, L.; Henkel, M.; Su, X.; Fang, X.; Wang, Q.; et al. Surfactin and fengycin contribute to the protection of a Bacillus subtilis strain against grape downy mildew by both direct effect and defence stimulation. Mol. Plant Pathol. 2019, 20, 1037-1050. [CrossRef] [PubMed]

29. Altschul, S.F.; Gish, W.; Miller, W.; Myers, E.W.; Lipman, D.J. Basic local alignment search tool. J. Mol. Biol. 1990, 215, 403-410. [CrossRef]

30. Hohenberg, P.; Kohn, W. Inhomogeneous Electron Gas. Phys. Rev. 1964, 136, B864-B871. [CrossRef]

31. Kohn, W.; Sham, L.J. Self-Consistent Equations Including Exchange and Correlation Effects. Phys. Rev. 1965, 140, A1133-A1138. [CrossRef]

32. Stewart, J.J.P. An investigation into the applicability of the semiempirical method PM7 for modeling the catalytic mechanism in the enzyme chymotrypsin. J. Mol. Model. 2017, 23, 154. [CrossRef] [PubMed]

33. Tirado-Rives, J.; Jorgensen, W.L. Performance of B3LYP Density Functional Methods for a Large Set of Organic Molecules. J. Chem. Theory Comput. 2008, 4, 297-306. [CrossRef] [PubMed]

34. Rassolov, V.A.; Ratner, M.A.; Pople, J.A.; Redfern, P.C.; Curtiss, L.A. 6-31G* basis set for third-row atoms. J. Comput. Chem. 2001, 22, 976-984. [CrossRef]

35. Trott, O.; Olson, A.J. AutoDock Vina: Improving the speed and accuracy of docking with a new scoring function, efficient optimization, and multithreading. J. Comput. Chem. 2010, 31, 455-461. [CrossRef] [PubMed]

36. Abraham, M.J.; Murtola, T.; Schulz, R.; Páll, S.; Smith, J.C.; Hess, B.; Lindahl, E. GROMACS: High performance molecular simulations through multi-level parallelism from laptops to supercomputers. SoftwareX 2015, 1, 19-25. [CrossRef]

Sample Availability: Samples of the compounds are not available from the authors.

(C) 2019 by the authors. Licensee MDPI, Basel, Switzerland. This article is an open access article distributed under the terms and conditions of the Creative Commons Attribution (CC BY) license (http://creativecommons.org/licenses/by/4.0/). 